Instituto Internacional de Investigación y Desarrollo Tecnológico Educativo INDTEC, C.A.

DOI: https://doi.org/10.29394/Scientific.issn.2542-2987.2021.6.19.14.279-294

OAI-PMH: http://www.indteca.com/ojs/index.php/Revista Scientific/oai

Ensayo Original / Original Essay

\title{
La Educación en un Mundo sin Fronteras
}

\author{
Autora: Belkys Rojas de Escalona \\ Universidad Nacional Experimental de las Fuerzas Armadas, UNEFA \\ belev16@gmail.com \\ Caracas, Venezuela \\ https://orcid.org/0000-0002-1185-0081
}

\section{Resumen}

El ensayo diserta acerca de la educación inmersa en la dinámica del mundo actual, el cual revela nuevas necesidades y exigencias en el diario acontecer. Cambios permanentes que obligan a la toma de consciencia en torno a la transitoriedad de la información, la complejidad del conocimiento y la volatilidad de la vida. El propósito se orienta a la generación de reflexiones en relación con este tema. En las actuales circunstancias, la educación juega, todavía, un papel fundamental; las nuevas tecnologías, la Inteligencia Artificial, la sociedad virtual, en general, promueven una educación ubicua, flexible, vinculada a la diversidad de la realidad, inclusiva, motivante y motivadora, orientada hacia la innovación permanente desde una evaluación critica, reflexiva y constructiva. Una educación cimentada en principios éticos: tolerancia, respeto, honestidad, equidad, justicia, igualdad, colaboración, responsabilidad, paz y la creatividad como eje de la acción didáctica.

Palabras clave: educación; mundo digital; sociedad virtual; valores.

Cómo citar este ensayo:

Rojas, B. (2021). La Educación en un Mundo sin Fronteras. Revista Scientific, 6(19), 279-294, eISSN: 2542-2987. Recuperado de: https://doi.org/10.29394/Scientific.issn.2542-

Fecha de Recepción: 27-07-2020
Fecha de Aceptación: $15-12-2020$
Fecha de Publicación: 05-02-2021 


\title{
Education in a World without Borders
}

\begin{abstract}
The essay talks about education immersed in the dynamics of the current world, which reveals new needs and demands in daily events. Permanent changes that force awareness of the transience of information, the complexity of knowledge and the volatility of life. The purpose is aimed at generating reflections in relation to this topic. In the current circumstances, education still plays a fundamental role; New technologies, Artificial Intelligence, virtual society, in general, promote a ubiquitous, flexible education, linked to the diversity of reality, inclusive, motivating and motivating, oriented towards permanent innovation from a critical, reflective and constructive evaluation. An education based on ethical principles: tolerance, respect, honesty, equity, justice, equality, collaboration, responsibility, peace and creativity as the axis of didactic action.
\end{abstract}

Keywords: education; digital world; virtual society; values.

How to cite this essay:

Rojas, B. (2021). Education in a World without Borders. Revista Scientific, 6(19), 279-294, e-ISSN: 2542-2987. Recovered from: https://doi.org/10.29394/Scientific.issn.2542-2987.2021.6.19.14.279$\underline{294}$

Date Received:

27-07-2020
Date Acceptance:

$15-12-2020$
Date Publication: 05-02-2021 


\section{Ensayo Original / Original Essay}

\section{Introducción}

Las nuevas tecnologías se constituyen en herramientas que contribuyen a generar espacios y momentos de reflexión orientados a la construcción dialéctica del aprendizaje de todos los participantes en el proceso. No se trata, de ofrecer cierta información a unos sujetos para que éstos la procesen y emitan una respuesta previamente determinada. En la nueva concepción del proceso educativo, el docente juega el roll de motivador a partir de la indagación y presentación de retos que promuevan la curiosidad y el interés de todos los participantes en la búsqueda de nuevas visiones de la realidad objeto de estudio en las diversas disciplinas.

Desde esta concepción de la educación en un mundo abierto y cosmopolita, todos, docentes y discentes, conforman un equipo de aprendizaje en el marco de un currículo fluido, flexible, dinámico, en construcción permanente. Tal como afirman Delgado, Urgilés y Vega (2020): la Generación $Z$ debe enfocarse en el desarrollo de competencias digitales ante las exigencias de un mundo globalizado.

La tecnología representa todo un potencial para cambiar la educación desde múltiples perspectivas. Sin embargo, el desarrollo fructífero e innovador depende de muchos factores. En primer lugar, es indispensable la alfabetización digital en todos los niveles del sistema, tanto en docentes como en estudiantes. La educación digital es un eje transversal de todo el proceso educativo, y no debe presentarse en asignaturas aisladas.

Los actores del proceso educativo, no sólo deben conocer el uso instrumental de la tecnología, sino, además, el impacto social de las mismas para generar el mayor provecho posible de su uso. No basta con el equipamiento tecnológico y la infraestructura necesaria para promover el cambio educativo en el marco de las exigencias socioculturales actuales. La educación digital comporta otra manera de concebir la realidad, de acercarse a ella para comprenderla y generar nuevos y mejores realidades. 
El presente ensayo responde a una inquietud académica producto de la dinámica de los tiempos actuales caracterizada por la avalancha de información permanente a través de múltiples vías y herramientas de comunicación. En ese orden de ideas, se revisan y reflexiona en torno a diferentes perspectivas planteadas por estudiosos del tema en el área específica de la educación para, a partir de la organización de las ideas, disertar y generar opiniones orientadas a incentivar el conocimiento, la comprensión y la búsqueda de nuevas vías hacia el futuro de la educación.

\section{Desarrollo}

\subsection{La Educación en la era digital}

En la era digital el acceso a la información es de tal magnitud, a través de las múltiples vías de acceso, que una de las competencias necesarias a desarrollar por el ente social es la búsqueda efectiva y eficaz de la información. No se trata entonces de encontrar datos, es necesario generar procesos reflexivos y creativos que coadyuven en la generación del pensamiento creativo e innovador. En la sociedad de la hiperinformación la educación debe enfocarse en la formación de individualidades conscientes de su poder crítico, reflexivos proclives al cambio, más allá de sujetos adaptados, repetidores, manejables.

Todo tiene un lado positivo y uno negativo y la comunicación digital no escapa a ello. La toxicidad de la comunicación se ha exacerbado en el marco de la proliferación de la información por lo que se hace necesario adquirir consciencia de este fenómeno para, sin caer en la paranoia de la incredulidad informativa, desarrollar un tamiz crítico y reflexivo que nos permita acceder a las múltiples aristas de la compleja realidad.

En la educación actual, el docente debe adecuar los recursos que ofrece la tecnología a los requerimientos educativos. Facebook y Twitter, por ejemplo, resultan muy útiles para fines didácticos. Así como también las 
plataformas colaborativas tales como: Google Classroom, Wikispaces, Genius, Skype, Hangouts, Chalkup, Zoom, Jitsi, etc, las cuales facilitan los procesos de enseñanza y aprendizaje a través de internet. y también, la gamificación que permite involucrar el juego al aprendizaje. Por otro lado, se han desarrollado muchas revistas digitales que promueven la publicación de artículos y documentos las cuales coadyuvan en la formación académica y en el avance científico y tecnológico.

El alumno en esta concepción de la educación, deja de ser un receptor de información que debe memorizar y repetir, para convertirse ahora en un creador de conocimiento, un protagonista activo de su propia formación integral: conocimientos, competencias, valores, actitudes. En este orden de ideas, los contenidos curriculares se orientan hacia el desarrollo de esa formación integral y la evaluación hacia el logro de las competencias requeridas. Los contenidos no son un fin en sí mismo, son medios para alcanzar el desarrollo de múltiples competencias.

Las experiencias didácticas con Realidad Aumentada (RA), por ejemplo, también han despertado el interés por esta tecnología. Estudios desarrollados en diferentes ámbitos internacionales por autores como Cabero y Barroso (2016); Montecé-Mosquera, Verdesoto-Arguello, MontecéMosquera y Caicedo-Camposano (2017): evidencian los altos niveles de satisfacción y motivación generados en escenarios formativos a partir de la praxis educativa con RA, en la cual los estudiantes pueden acceder a contenidos virtuales en tercera dimensión con tecnología móvil de bajo costo.

En este sentido, García (2017a): alude a las tendencias tecnológicas planteadas en el Informe Horizon Report por Adams, Cummins, Davis, Freeman, Hall y Ananthanarayanan (2017): las cuales se pondrán en vigencia en menos de un año, con el Aprendizaje Adaptativo y Aprendizaje Móvil.

El Aprendizaje Adaptativo: se revela como uno de los mayores adelantos de la época. Desde esta perspectiva, la acción docente, los recursos 


\section{Ensayo Original / Original Essay}

y estrategias se orientan hacia la satisfacción de las necesidades específicas de aprendizaje de cada sujeto. Las tecnologías adaptativas captan la información de los Big Data individuales y grupales para facilitar el desarrollo de estrategias docentes adecuadas a las características de grupos y alumnos concretos.

Por otro lado, el Aprendizaje Móvil: cada día más asequible al público a través de los múltiples dispositivos: tablets, teléfonos inteligentes, relojes, entre otros, que abren más y mejores posibilidades a la comunicación en todos sus ámbitos, incluyendo por supuesto a la educación. En esa misma línea, van't Hooft, Swan, Cook y Lin (2007): afirman que este tipo de aprendizaje, denominado Aprendizaje Ubicuo, promueve el uso de cualquier dispositivo, no sólo el móvil, lo cual amplia las posibilidades de acceso comunicacional en términos de espacio y tiempo. Esta concepción de aprendizaje está en sintonía con el enfoque del Aprendizaje situado (Situated learning) desarrollada por Lave y Wenger (1991): el cual sostiene que el verdadero aprendizaje se construye en el marco de una relación directa con la realidad objeto de estudio por lo que promueve actividades contextualizadas.

El Informe de la Organización de las Naciones Unidas para la Educación, la Ciencia y la Cultura (UNESCO, 2013a): plantea a los políticos responsables de las decisiones en el ámbito educativo la necesidad de orientar a los actores del proceso educativo con relación al fomento del uso del aprendizaje a través del móvil y cómo aprovecharlo para mejorar la educación. En referencia al tema, García (2017b), resume los beneficios potenciales de estas tecnologías, planteados en el Informe de la UNESCO (2013b):

Ampliar el alcance y la equidad de la educación; Facilitar el aprendizaje personalizado; Proporcionar feedback y evaluación inmediatos; Habilitar el aprendizaje en cualquier momento y en cualquier lugar; Asegurar un uso más productivo del tiempo que pasamos en clase (flipped classroom); Crear nuevas comunidades de aprendizaje; Apoyar el aprendizaje situado (realidad aumentada); Facilitar la continuidad de la 
experiencia de aprendizaje; Conectar el aprendizaje formal y el aprendizaje informal; Reducir la perturbación de la educación en casos de conflicto y desastres; Ayudar a los aprendices con discapacidades; Mejorar la comunicación y la administración; Minimizar los costes y maximizar la eficiencia (pág. 21).

El informe de la UNESCO (2013c), enfatiza el hecho de que la introducción de las tecnologías en educación no supone, por sí mismo, un mejoramiento del proceso educativo:

La región tiene todavía el desafío de una incorporación fecunda de las TICs a la enseñanza. Esto implica no solo elevar sus indicadores de acceso, sino capacitar mejor a sus docentes, y elaborar programas y diseños curriculares que utilicen intensivamente las nuevas tecnologías (pág. 130).

Indiscutiblemente las nuevas tecnologías representan retos para la educación. La preparación de los docentes en competencias digitales, la dotación de los recursos tecnológicos para las instituciones educativas; la adaptación de los contenidos curriculares a las nuevas estrategias y recursos son sólo algunos de los desafíos de la nueva era digital.

\subsection{La evaluación en el proceso educativo, mediado por la tecnología}

La evaluación es un aspecto fundamental en el proceso educativo, tanto desde una perspectiva diagnóstica como formativa y sumativa; debe ofrecer información relevante que conduzca tanto al logro de los objetivos y propósitos previstos como a la mejora continua del proceso. Se han desarrollado softwares de valoración que permiten recabar los datos provenientes de exámenes y diversas pruebas orientadas a calificar el progreso de los estudiantes. Los videos interactivos, entrevistas virtuales, Portafolios, Foros, Rubricas y muchas herramientas tecnológicas más pueden coadyuvar en el proceso evaluativo para ir más allá de medir conocimientos y habilidades.

En el proceso evaluativo es fundamental la individualización, no todos 
los alumnos aprenden de la misma manera y al mismo tiempo. La tecnología abre posibilidades para adecuar el proceso a los diferentes ritmos de aprendizaje. Una evaluación continua, diversa, motivante, personalizada. Una evaluación promotora del cambio social a través de la innovación. El proceso educativo online, permite la evaluación reflexiva tanto personalizada como grupal y, además abre vías para compartir experiencias acerca del proceso educativo y evaluativo, desarrollando una interpretación crítica y reflexiva de los procesos vividos en el acontecer educativo con miras a generar cambios motivados y motivadores.

La evaluación como parte fundamental del proceso educativo, debe promover el desarrollo del pensamiento reflexivo. Desde esta perspectiva, el facilitador propondrá actividades evaluativas orientadas al análisis, a la apertura a la diversidad de visiones y alternativas que conduzcan al estudiante a pensar más allá de sus expectativas y conocimientos previos. Se busca estimular el pensamiento crítico y creativo con miras a la formación de un ser consciente de sus potencialidades capaz de generar los cambios que la sociedad reclama.

Más allá de una evaluación centrada en medir el nivel de conocimientos adquiridos en una determinada asignatura, la evaluación debe orientarse hacia la evaluación personal de las competencias duras y blandas que el sujeto desarrolla en el marco de la realidad espaciotemporal en la que le tocará desenvolverse. En este orden de ideas, cobra relevancia la retroalimentación expresada a través de la coevaluación desarrollada en un proceso dialéctico, abierto, reflexivo orientado hacia al reconocimiento de nuevas perspectivas.

Por supuesto, la autoevaluación también juega un papel fundamental en el proceso educativo dado que sólo es posible el cambio cognitivo-afectivo y social a partir de la toma de consciencia y de la interacción con los otros. El docente debe planificar el proceso evaluativo en sintonía con las estrategias didácticas. Las actividades evaluativas, más que una forma de juzgar, deben 
ser concebidas como una vía para el aprendizaje tanto del estudiante como del docente. La evaluación representa una forma de crecimiento personal, académico e institucional en la que deben estar inmersos todos los implicados en el proceso con miras a generar reflexión y cambio con miras a optimizar personas, procesos y por ende sociedad.

El proceso evaluativo debe estar fundamentado en el respeto mutuo y la apertura hacia un aprendizaje dialéctico en el cual, el docente como facilitador:

- Proponga preguntas que generen reflexión.

- Permita profundizar en aspectos relevantes.

- Destaque los logros del estudiante.

- Promueva la autoidentificación de las competencias de los implicados en el proceso.

- Favorezca la construcción del aprendizaje de todos los implicados.

\subsection{La Creatividad: puntal de la nueva era de un mundo sin fronteras}

En la era de la Inteligencia Artificial, la creatividad representa el motor y el norte de la acción humana. El desarrollo de la tecnología y su presencia obligada en todos ámbitos de la vida personal y social harán imperativo la formación de competencias en el área digital de todos los miembros de la sociedad. De manera que el algoritmo será un componente obligado en el proceso creativo. Más que desplazar la creatividad humana, la IA será el detonante que impulsará su desarrollo. En esa misma línea, Boden (1994), distingue tres tipos de creatividad y sostiene que los ordenadores son capaces de evidenciar los tres:

- Creatividad combinatoria: entendida como la capacidad para asociar ideas conocidas y transformarlas en algo novedoso.

- Creatividad exploratoria: consiste en ejecutar acciones concretas para 


\section{Ensayo Original / Original Essay}

la realización de una idea hasta entonces inimaginable.

- Creatividad transformadora: se refiere a transformar algo ya existente para producir otro concepto.

La tecnología en sus múltiples expresiones representa una herramienta orientada a impulsar el desarrollo creativo del ser humano. En conformidad con Ortega (1914): el ser humano es él y su circunstancia. El momento actual representa un reto en la evolución del ser humano, es su imaginación, experiencia y conocimientos lo que alimenta la máquina para generar progreso y bienestar al planeta. Tal vez en algún momento la IA equipare o supere la inteligencia humana lo cual conducirá a que el humano deje de ocupar su tiempo en actividades repetitivas y tediosas para dedicarse al desarrollo innovador de la humanidad.

Obviamente la educación tendrá que adecuarse a los nuevos tiempos, nuevas formas de aprendizaje y enseñanza, que reclaman preparación y roles diferentes para el docente y la institución educativa en general, una educación centrada en:

- Aprendizaje personalizado fundamentado en las características individuales de cada sujeto.

- Mayor aprovechamiento del potencial humano.

- Valoración de la diversidad.

- Énfasis en la inclusión y la interactividad.

Las estrategias didácticas deben estar orientadas a promover el disfrute del proceso más que la expectativa por el resultado, más allá de aprobar una asignatura, la dinámica educativa ha de generar retos que motiven al sujeto a una relación dialéctica y empática con el entorno. Aprender a percibir e interpretar las múltiples facetas o caras de una situación particular incide en el 
desarrollo de una mentalidad abierta a nuevas posibilidades, lo cual representa un requisito del pensamiento disruptivo, propio de la nueva era. La educación ha de cambiar su orientación centrada en la rutina, la conformidad y los convencionalismos hacia una visión dinámica, orientada al desarrollo personal y al compromiso planetario.

Ante las exigencias de mundo actual, la educación tiene que integrar lo didáctico con lo tecnológico en un proceso de enseñanza y aprendizaje orientado hacia el desarrollo de habilidades tanto blandas como duras. Es decir, la educación no sólo debe velar por la formación en competencias técnicas en áreas específicas del conocimiento. También es necesario, fomentar el desarrollo personal e interpersonal hacia la creatividad, resiliencia y proactividad.

Al respecto, Casanova (2012): considera tres ejes imprescindibles para que la educación basada en competencias se convierta en una realidad más allá del discurso vacío: trabajo en equipo, planteamiento interdisciplinar de las materias curriculares y transversalidad de las competencias. Destaca la necesidad de una estrecha conexión transversal tanto entre los contenidos de las diferentes áreas del conocimiento como de las competencias que se pretende formar. A fin de gestionar una formación integral para un sujeto capaz de interactuar de manera ética, consciente, hábil, reflexiva y creativa en el desarrollo de un mundo mejor. En ese marco, la tecnología representa un recurso que abre posibilidades al diálogo abierto, creativo y productivo.

\subsection{Resultados del proceso auto y hetero formativo: Valores en la Educación Virtual}

Los entornos virtuales por su condición de espacios para el intercambio social tienen una fuerte carga de emociones, sensaciones, perspectivas impregnadas de valores. De ahí que el docente como gestor del proceso educativo debe generar las condiciones para la construcción de relaciones 


\section{Ensayo Original / Original Essay}

basadas en el respeto, tolerancia, reconocimiento del otro, responsabilidad, colaboración, participación.

La dinámica social actual demanda pensamiento y acción permanente hacia una educación axiológica que oriente la vida de los ciudadanos del mundo hacia horizontes de progreso fundamentados en la paz, la armonía, la fraternidad más allá de intereses meramente económicos. El docente juega un roll fundamental en esta relevante tarea. En el marco de la nueva praxis didáctica, es indispensable la acción reflexiva y consciente del docente, orientada hacia la construcción de los valores. Desde esta perspectiva, la planificación, diseño y aplicación de estrategias didácticas debe orientarse a:

- Promover la libre expresión del estudiante, respetando y estimulando la diversidad de puntos de vista, piedra angular del pensamiento creativo.

- Generar la participación en actividades colaborativas que favorezcan el disfrute del compartir.

- Estimular la responsabilidad como guía de las acciones propias y ejemplo para las ajenas. La entrega puntual de las asignaciones, asistencia a los eventos programados.

- Favorecer el aprendizaje activo y autónomo fundado en el reconocimiento de las competencias propias que llevan al estudiante a la valoración personal y al reconocimiento y respeto por los logros ajenos.

Se conjuga entonces, en este proceso la auto formación entendida como la toma de consciencia del propio ser, de sus habilidades, conocimientos y potencialidades de cara al desarrollo personal, conjuntamente con el reconocimiento del otro y del entorno social, ambiental, tecnológico con miras al desarrollo de un mundo centrado en la convivencia. Ante esto, Duart (2002): afirma: 
Hablar de educación en valores no es hablar de didácticas específicas o de técnicas para fomentar determinadas maneras de ser o de actuar. Hablar de educación en valores es hablar de la necesidad de crear y de favorecer espacios de vivencia en los que las personas podamos sentir, experimentar, vivenciar algo que rompa nuestra indiferencia, algo que nos emocione e impacte en nuestro interior (pág. 59).

\section{A manera de Conclusión}

El mundo en su rápido y multifacético acontecer genera cambios en todas las áreas: económica, política, cultural, social. En el marco de esta dinámica, la educación, la cual sólo ha recibido, en la práctica, cambios poco profundos a lo largo de la historia, se ve obligada a afrontar grandes desafíos. En pocas palabras, o la educación cambia radicalmente para volverse más flexible, abierta, promotora de la autogestión, la creatividad y la innovación o simplemente desaparecerá como promotora del cambio social.

En este mundo sin fronteras, el ser humano debe formarse con una mirada abierta a la diversidad que le permita comprender las múltiples expresiones del acontecer global. La educación ha de estar orientada a la formación de competencias para una vida plena y satisfactoria. El reto implica salir de los paradigmas tradicionales generando modelos educativos que ofrezcan respuestas a la realidad disruptiva actual y futura. En este marco, la tecnología tiene un papel protagónico como herramienta para el impulso de la innovación educativa.

\section{Referencias}

Adams, S., Cummins, M., Davis, A., Freeman, A., Hall, C., \& Ananthanarayanan, V. (2017). NMC Horizon Report: 2017 Higher Education Edition. ISBN: 978-0-9977215-7-7. Estados Unidos: New Media Consortium. Recuperado de: https://eric.ed.gov/?id=ED582134

Boden, M. (1994). La mente creativa: Mitos y mecanismos. ISBN: 84-7432- 
500-5. Barcelona, España: Editorial Gedisa.

Cabero, J., \& Barroso, J. (2016). Posibilidades educativas de la Realidad Aumentada. NAER. New Approaches in Educational Research, 5(1), 46-52, e-ISSN: 2254-7399. Recuperado de:

http://dx.doi.org/10.7821/naer.2016.1.140

Casanova, M. (2012). El Diseño Curricular como Factor de Calidad Educativa. Revista Iberoamericana sobre Calidad, Eficacia y Cambio en Educación, 10(4), 7-20, e-ISSN: 1696-4713. Recuperado de: https://revistas.uam.es/reice/article/view/2984

Delgado, D., Urgilés, D., \& Vega, P. (2020). X-Y. Ahora vienen los Z: Una Generación de nuevos Ziudadanos. Revista Scientific, 5(16), 290304, e-ISSN: 2542-2987. Recuperado de:

https://doi.org/10.29394/Scientific.issn.2542-2987.2020.5.16.15.290$\underline{304}$

Duart, J. (2002). Educar en valores en entornos virtuales de aprendizaje: realidades y mitos. Apertura, (2), 58-64, ISSN: 1665-6180. Universidad de Guadalajara: Coordinación de Recursos Informativos del Sistema de Universidad Virtual.

García, L. (2017a,b). Educación a distancia y virtual: calidad, disrupción, aprendizajes adaptativo y móvil. RIED. Revista Iberoamericana de Educación a Distancia, 20(2), 9-25, ISSN: 1138-2783; e-ISSN: 13903306. Recuperado de: http://dx.doi.org/10.5944/ried.20.2.18737

Lave, J., \& Wenger, E. (1991). Situated learning: Legitimate peripheral participation. ISBN: 9780511815355. United States: Cambridge University Press.

Montecé-Mosquera, F., Verdesoto-Arguello, A., Montecé-Mosquera, C., \& Caicedo-Camposano, C. (2017). Impacto de la Realidad Aumentada en la Educación del Siglo XXI. European Scientific Journal, 13(25), 129-137, ISSN: 1857-7881; e-ISSN: 1857-7431. Recuperado de: 
http://dx.doi.org/10.19044/esj.2017.v13n25p129

Ortega, J. (1914). Meditaciones del Quijote. Madrid, España: Residencia de Estudiantes.

UNESCO (2013a,b,c). Situación Educativa de América Latina y el Caribe: Hacia la educación de calidad para todos al 2015. Chile: Oficina Regional de Educación para América Latina y el Caribe (OREALC/UNESCO Santiago).

van't Hooft, M., Swan, K., Cook, D., \& Lin, Y. (2007). What is ubiquitous computing?. In M. van 't Hooft \& K. Swan (Eds.), Ubiquitous computing in education: Invisible technology, visible impact (pp. 3-17). Mahwah, NJ, United States: Lawrence Erlbaum \& Associates. 


\section{Belkys Rojas de Escalona \\ e-mail: belev16@gmail.com}

Nacida en Caracas, Venezuela, el 12 de octubre del año

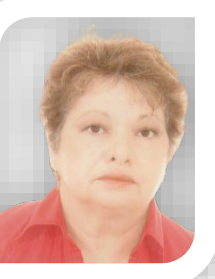
1974. Doctorado en Ciencias de la Educación por la Universidad Central de Venezuela (UCV); Coordinadora del Programa Doctorado en Innovaciones Educativas de la Universidad Nacional Experimental Politécnica de la Fuerza Armada (UNEFA), desde el año 2006; Profesora Titular jubilada de la Universidad Pedagógica Experimental Libertador (UPEL, 2001); Autora de libros como: La resiliencia en la organización universitaria: Un reto hacia el futuro, publicado por la Editorial Académica Española en el año 2011; Investigación cualitativa: Fundamentos y praxis, publicado por la Editorial de la Universidad Pedagógica Experimental Libertador (FEDUPEL, 2007, 2010, 2014) en Caracas; Hacia una evaluación de la creatividad en preescolar por la (FEDUPEL, 2004); y Artículos relacionados con el área de la creatividad en la organización, la creatividad infantil y la investigación cualitativa.

El contenido de este manuscrito se difunde bajo una Licencia de Creative Commons ReconocimientoNoComercial-Compartirlgual 4.0 Internacional 